\title{
Les espaces de débat comme méthodologie d'intervention capacitante pour enrichir le retour d'expérience
}

Work debate spaces as an intervention method for developing capabilities and for enriching learning from experience

\section{Christelle Casse et Sandrine Caroly}

\section{Q OpenEdition}

Journals

Édition électronique

URL : http://journals.openedition.org/activites/3008

DOI : 10.4000/activites.3008

ISSN : 1765-2723

Éditeur

ARPACT - Association Recherches et Pratiques sur les ACTivités

Référence électronique

Christelle Casse et Sandrine Caroly, « Les espaces de débat comme méthodologie d'intervention capacitante pour enrichir le retour d'expérience », Activités [En ligne], 14-2 | 2017, mis en ligne le 15 octobre 2017, consulté le 19 avril 2019. URL : http://journals.openedition.org/activites/3008; DOI : 10.4000/activites.3008

Ce document a été généré automatiquement le 19 avril 2019

\section{(i) $\Theta \Theta$}

Activités est mis à disposition selon les termes de la licence Creative Commons Attribution - Pas d'Utilisation Commerciale - Pas de Modification 4.0 International. 


\section{Les espaces de débat comme méthodologie d'intervention capacitante pour enrichir le retour d'expérience}

Work debate spaces as an intervention method for developing capabilities and for enriching learning from experience

Christelle Casse et Sandrine Caroly

\section{NOTE DE L'ÉDITEUR}

Article soumis le 22/07/2016, accepté le 22/07/2017

\section{Introduction}

1 Face à la complexité des systèmes productifs actuels et aux exigences d'adaptation qu'imposent les marchés, les entreprises doivent de plus en plus s'appuyer sur les compétences et la capacité des salariés à gérer les incidents dans le travail. Ces préoccupations rejoignent celles du législateur qui promeut, à travers la réglementation du travail, l'ouverture du dialogue dans les entreprises et la mise en place d'espaces de débat sur le travail pour donner la parole aux acteurs de terrain, développer la confrontation des points de vue comme moyen de faire face à la complexité, d'être plus efficace et sécuritaire.

2 Les ergonomes revendiquent depuis de nombreuses années l'importance de la participation de tous les acteurs et en particulier des opérateurs de terrain dans les projets de transformation organisationnelle ou spatiale dans les entreprises. Cependant, les approches évoluent, passant d'approches plutôt défensives (des conditions de travail) 
et réactives, à des approches constructives (Falzon, 2013) et pro-actives. L'ergonome contribue non seulement à concevoir des systèmes de travail qui ne soient pas délétères pour la santé des travailleurs, mais il se préoccupe aussi de plus en plus de mettre en place les conditions de développement des individus ainsi que de l'organisation. La temporalité dans laquelle il inscrit son action évolue ainsi sur le long terme, dépassant le cadre formel de l'intervention. Son objectif principal devient la conception d'organisations capacitantes (Arnoud \& falzon, 2013a; Sen, 1993) c'est-à-dire qui portent en elles les facteurs de développement potentiel des capacités futures des acteurs et qui favorisent l'instauration d'une dynamique d'apprentissage organisationnel.

Ces nouvelles exigences ont des répercussions sur les méthodes d'intervention déployées par les ergonomes, qu'ils soient chercheurs ou praticiens. Un certain nombre de recherches et de publications font état aujourd'hui de ces nouvelles approches de l'intervention et des méthodes qu'elles mobilisent. Cependant, peu de modèles sont disponibles à ce jour pour formaliser ces nouvelles méthodes. L'objectif de cet article est d'analyser le déroulement d'une intervention ergonomique visant à enrichir un dispositif de retour d'expérience (REX) chez un exploitant de tunnels routiers, pour mettre en lumière et discuter les apports des espaces de débat comme méthode d'intervention capacitante pour enrichir le retour d'expérience.

4 Nous verrons dans un premier temps l'évolution des méthodes de l'intervention en ergonomie et les modèles sur les espaces de débat sur le travail. Dans un second temps, nous appuyant sur une mise en récit structurée de l'intervention sur le REX, nous analyserons les composantes et le déroulement de cette intervention à visée capacitante. Nous discuterons enfin des apports de cette analyse concernant la modélisation et les conditions d'une intervention capacitante et les évolutions qu'elle induit sur le métier des intervenants en ergonomie.

\section{1. Évolution des approches de l'intervention ergonomique : de la démarche projet à l'approche capacitante}

\subsection{La démarche projet en ergonomie}

5 L'intervention ergonomique a traditionnellement pour visée l'amélioration des situations de travail en intégrant les objectifs de santé des travailleurs et de performances des entreprises, sur les plans à la fois de la qualité et de la sécurité. Selon Petit (2005), l'objectif de l'intervention ergonomique est le développement conjoint de la structure organisationnelle et des activités humaines, des interactions entre les acteurs de l'entreprise. Du point de vue des méthodes, l'intervention s'appuie sur une démarche de conduite de projet inspirée des démarches de conception développées dans le champ de l'ingénierie. Cette démarche met l'accent sur deux phases clés de l'intervention : d'une part elle valorise le diagnostic qui sera partagé avec les acteurs afin de confronter les représentations et construire une représentation partagée. D’autre part, elle est aussi centrée sur la démarche participative de conception ad hoc, qui débouche sur un livrable exprimé par une nouvelle prescription, qui clôture l'intervention. Cette démarche, largement traitée et documentée aujourd'hui par les chercheurs en 
ergonomie, est synthétisée par Barcellini, Van Belleghen et Daniellou (2013) dans un modèle qui découpe l'intervention en trois phases essentielles :

1. une phase d'analyse de l'activité existante pour construire et choisir des situations de références à transposer dans le futur, qui serviront à la construction de scénarios de prescriptions.

2. une phase de co-conception basée sur la simulation des scénarios de prescriptions, engageant les acteurs de l'entreprise, opérateurs et managers, futurs «acteurs» de la nouvelle organisation, afin de croiser les points de vue et les contraintes (Garrigou, Thibault, Jackson, \& Mascia, 2001) ;

3. une phase d'expérimentation des nouvelles prescriptions et éventuellement d'ajustement, dans laquelle une activité nouvelle va se déployer. C'est aussi la phase de pérennisation du dispositif conçu.

6 Le rôle de l'intervenant dans ce type d'intervention est de faire l'analyse du travail et de construire un diagnostic qui sera restitué et partagé avec les acteurs de l'entreprise, puis d'organiser et d'accompagner la démarche participative de co-conception, notamment à l'aide de supports tels que la simulation spatiale ou organisationnelle, et enfin de réaliser les ajustements nécessaires une fois la nouvelle prescription prise en main par les acteurs (Petit, 2005).

7 Cette démarche d'intervention développée depuis une trentaine d'années par les praticiens en ergonomie a produit des résultats tout à fait intéressants pour concevoir et transformer des systèmes de travail qui améliorent les conditions de travail et la performance. Cependant, cette démarche présente des limites du point de vue du développement à long terme des individus et de l'organisation, que les approches constructives développées récemment visent à dépasser pour « développer le potentiel capacitant des organisations » (Falzon, 2013, p. 2).

\subsection{L'intervention capacitante visant le développement des individus et de l'organisation}

8 L'approche constructive de l'ergonomie (Falzon, 2013) a pour objectif le développement des personnes et de l'organisation. Dans cette perspective, la visée de l'intervention «consiste à concevoir non pas une nouvelle organisation, mais un dispositif d'expérimentation et d'apprentissage, pour susciter et favoriser de nouveaux fonctionnements organisationnels» (Sardas \& Lefevre in Arnoud \& Falzon, 2013b). D'après Mollo et Nascimento (2013), pour favoriser le développement, l'intervention doit permettre de concevoir une organisation « débattable " (ibid, p. 233), dont les règles et les principes peuvent être discutés par les acteurs. L'objectif de l'intervention dépasse la temporalité de la définition du projet pour s'inscrire dans un temps plus long, à l'échelle de la vie quotidienne de l'organisation. Les méthodes d'intervention doivent alors évoluer, passant de méthodes de conception à des méthodes de formation et d'expérimentation collective (Van Belleghem, 2016). Les espaces de débat sur le travail constituent l'une des formes promues par les chercheurs et les praticiens en ergonomie (Casse, 2015 ; Rocha, Mollo, \& Daniellou, 2015 ; Van Belleghem \& Fortiolli Conti, 2015), mais aussi dans d'autres disciplines telles que la clinique de l'activité (Clot, Faïta, Fernandez, \& Scheller, 2000 ; Clot, 2006) ou les sciences de gestion (Detchessahar, 2013 ; Detchessahar, Gentil, Grevin, \& Stimmec, 2015), pour favoriser le développement de 
l'organisation et des individus du point de vue de leurs compétences, de leur bien-être et de leur santé.

\subsubsection{Les espaces de débat sur le travail : une méthode d'intervention capacitante}

L'intervention capacitante a pour objectif de détecter et construire les ressources des acteurs en matière de développement, ainsi que les facteurs qui facilitent (ou inhibent) la conversion des ressources en possibilités effectives (Arnoud, 2013; Sen, 1993). Rocha (2014) a montré que la mise en place d'espaces de débat sur le travail constitue une ressource pour le développement des acteurs et de l'organisation, car cela permet de développer les apprentissages croisés entre les individus, ainsi que les capacités individuelles et collectives à agir, de résoudre les problèmes récurrents en construisant des solutions d'amélioration pérennes.

Detchessahar (2001) propose de créer des espaces de débat structurés, institutionnalisés et protégés qui visent principalement à réaliser «l'ensemble des arrangements, compromis et bricolages que supposent l'incomplétude de la prescription et le caractère irréductiblement erratique de l'activité concrète » (Detchessahar, 2013, p. 59).

11 Toutefois, selon les chercheurs, pour que le débat sur le travail soit constructif et qu'il aboutisse à des transformations concrètes des règles et de l'organisation qui portent le développement, plusieurs caractéristiques doivent être réunies (Detchessahar, 2011).

- Le débat doit être centré sur le travail, les arbitrages et les régulations quotidiennes. Pourtant, accéder au travail réel pour le mettre en débat n'est pas un processus spontané. Le risque qu'elle dérive sur des problèmes généraux qui n'apporteraient pas de transformation - ni personnelle, ni productive - est réel (Caroly, 2010), comme beaucoup de réunions d'équipe qui sont consacrées à la transmission unilatérale d'informations descendantes et de consignes. Le dévoilement de leur activité n'est pas sans risque pour les travailleurs (Dejours, 2009) ; par conséquent, cela peut générer chez eux des résistances ou des défenses qui empêchent tout débat sur le travail réel. Plusieurs méthodes existent, développées par les psychologues du travail et les ergonomes, pour convoquer l'activité (Van Belleghem, 2016) dans les débats. Elles ont pour objectif de permettre aux opérateurs de retraverser leur expérience de façon concrète: les faits, les processus de décisions et d'actions, la complexité d'une situation. L'objectif de ces méthodes est toujours d'éviter les discours sur l'expérience, faits d'opinions et de jugements, pour faire émerger au contraire le dire de l'expérience, au plus près de sa réalité matérielle, sensible et cognitive pour la personne. La simulation notamment est utilisée par les ergonomes pour convoquer l'activité dans les projets de conception, avec la particularité qu'il s'agit là non pas de convoquer une situation passée, mais de se projeter dans l'activité future liée à un contexte prescrit nouveau (Barcellini, Van Belleghem \& Daniellou, 2013 ; Maline, 1997 ; Rogalski, 1994, 1995), défini dans un scénario.

- Le débat est animé par un manager de proximité qui connaît le métier et pourra aider le groupe à avancer. Les espaces de débat ont aussi vocation à (re)donner aux managers de proximité une place centrale dans les processus de régulation en réinstallant « au cœur de sa fonction, l'activité de régulation et de soutien au travail réel »(Van Belleghem \& al, 2015).

- Le débat est régulier, fréquent et s'inscrit dans le long terme, pour instaurer la confiance, notamment entre les opérateurs et les managers (Mollo \& Nascimento, 2013). 
- Il est aussi outillé grâce à des outils de gestion qui permettent de suivre l'activité et d'éclairer les points de vue des participants. Et il laisse une trace, soit par une formalisation, soit au travers des solutions produites et des accords qui fondent le collectif.

12 Ces conditions permettent de créer des espaces de débat sécurisés et efficaces, qui contiennent la réflexion des acteurs sur leur travail (Chaudet, 2013 ; Hoyrup et Elkjaer, 2006) et aboutissent à des évolutions concrètes. L'ingénierie du débat fonctionne si elle s'insère dans un "écosystème organisationnel » (Detchessahar, 2013) qui soutient le débat. Cela signifie que l'organisation offre les conditions pour que les individus soient en capacité de discuter; des conditions favorisant l'engagement des managers et des autres acteurs dans la démarche (temps, marges de manœuvre, formation, évaluation), et des conditions concernant la structuration et l'articulation des espaces de débat entre eux.

Les modèles de l'intervention ergonomique ont donc évolué ces dernières années, passant d'une approche projet à une approche constructive, visant le développement des individus et de l'organisation, fondée sur la mise en place d'expérimentations de dispositifs innovants tels que les espaces de débat structurés sur le travail.

\subsubsection{L'intervention capacitante pour enrichir le retour d'expérience}

Dans le domaine de la maitrise des risques, l'approche capacitante présente plusieurs intérêts. Elle permet de dépasser une vision de la sécurité essentiellement basée sur la "sécurité réglée " et les règles formelles de l'organisation, pour s'intéresser au fonctionnement quotidien de l'organisation et des collectifs de travail comme ressource pour gérer la sécurité. Le REX vise à améliorer le futur en s'appuyant sur l'analyse des évènements passés. Il constitue aujourd'hui un des piliers des systèmes de management de la sécurité dans les grandes entreprises. Cependant les dispositifs de REX présentent aussi de nombreuses limites bien décrites dans la littérature, concernant notamment la prise en compte des "signaux faibles ", des situations critiques quotidiennes, des facteurs humains et organisationnels (Dechy \& Dien, 2007). Le REX est souvent perçu par les acteurs opérationnels comme un dispositif formel éloigné de la réalité et des préoccupations du terrain, souvent source de sanctions, qui peut pour ces raisons être déserté par les opérationnels. Face à une situation de silence organisationnel de ce type dans une entreprise de distribution d'électricité, Rocha (2014) a utilisé les espaces de débat comme une méthodologie pour structurer le REX sur des situations courantes dans des métiers de techniciens d'installation, travaillant de façon isolée. Cette rechercheintervention a abouti au développement du collectif de pairs, à la transformation des relations opérateurs-encadrement, à la construction de règles d'action efficaces et de solution techniques pérennes. Les espaces de débat sont apparus comme une méthode d'intervention capacitante permettant de partager sur les pratiques individuelles, de définir l'espace des pratiques acceptables 4et de trouver des solutions collectives aux difficultés qui émergent du terrain.

La question à laquelle notre article cherche à répondre est la suivante : comment les espaces de débat, en tant que méthode d'intervention capacitante, peuvent enrichir le retour d'expérience dans un contexte d'activité à risque et de collectif transverse? L'analyse réflexive et la mise en récit structurée d'une intervention à visée capacitante, menée chez un exploitant de tunnels routiers, doit contribuer à répondre à cette question. 


\section{Mise en récit d'une intervention capacitante visant l'enrichissement du REX par les espaces de débat}

\subsection{Une méthode d'analyse réflexive de l'intervention pour une mise en récit structurée}

Notre objectif d'une mise en récit de l'intervention est de contribuer à produire des connaissances sur la recherche sur la pratique de l'intervention en ergonomie, notamment sur les apports des espaces de débat structurés pour l'intervention capacitante et l'enrichissement du REX.

Les données présentées et analysées dans cet article ont été sélectionnées et consolidées a posteriori. Nous nous sommes appuyées sur un modèle proposé par Petit, Querelle \& Daniellou (2007) visant à structurer les données d'une intervention ergonomique pour la mettre en récit et produire des connaissances sur la pratique d'intervention.

Nous avons donc reconstitué 5 catégories de données, à partir des documents-traces et des résultats de l'intervention (ibid). Ces catégories de données sont les suivantes :

- les hypothèses et les buts de l'intervenant;

- la méthode et la stratégie d'implantation de l'intervention ;

- la dynamique réelle du processus d'intervention ;

- les retournements et les surprises ;

- les résultats et impacts de l'intervention.

Les données utilisées a posteriori sur l'intervention sont issues de trois sources principales :

- le document de recherche décrivant le contexte d'intervention, la méthodologie employée et les résultats et limites de l'intervention réalisée (Casse, 2015);

- les comptes-rendus de réunions du comité de suivi de la thèse, qui se sont déroulées à une fréquence de 6 par an environ pendant toute la durée de la thèse. Ces réunions réunissaient la direction de l'entreprise, les représentants du Centre d'Étude des Tunnels (CETU) et les universitaires. Elles avaient pour objectif de discuter des avancées de la recherche et de l'intervention et des propositions méthodologiques qui en émergeaient au fur et à mesure de son déroulement. Les comptes rendus de ces réunions portent les traces de l'évolution des choix méthodologiques, des résultats intermédiaires et des décisions des acteurs en lien avec ces éléments ;

- les comptes rendus de groupe de travail et de comité de pilotage durant la démarche participative de conception de la nouvelle organisation du REX.

La mise en récit de l'intervention met en évidence les éléments structurants de l'intervention, qui ont contribué aux résultats de l'intervention.

\subsection{Le contexte de l'intervention : un projet d'amélioration du REX en lien avec les pratiques de terrain}

21 Notre objet d'étude est une intervention menée dans le cadre d'un projet de recherche initié par le CETU portant sur l'amélioration du REX chez les exploitants de tunnels routiers pour mieux maitriser les risques. Menée en partenariat avec un exploitant de tunnel, cette intervention avait comme visée pratique la re-conception d'un dispositif de 
REX qui contribue au développement des pratiques de terrain et des compétences collectives en matière de gestion de la sécurité. Dans une approche capacitante, notre objectif était de mettre en place les bases d'un REX qui favorise le développement des individus et de l'organisation, en nous appuyant notamment sur la mise en place d'espaces de débat sur le travail.

L'entreprise d'exploitation partenaire de l'intervention est une filiale d'une société d'autoroute qui emploie une centaine de salariés pour gérer le trafic et la sécurité d'un périphérique urbain à péages de dix kilomètres, comportant quatre tunnels. Les tunnels sont des infrastructures complexes dotées d'un grand nombre d'équipements sophistiqués reliés à un système de Gestion Technique Centralisée pilotée depuis un PC de supervision. Grâce à ces équipements, les superviseurs peuvent surveiller en permanence l'ouvrage, gérer le trafic et les évènements. Les patrouilleurs font le petit entretien de l'ouvrage et interviennent lors des travaux ou en cas d'évènement, pour mettre en sécurité les usagers et le cas échéant favoriser l'intervention des secours. Des techniciens assurent la maintenance de l'ouvrage au quotidien et participent à la gestion technique des évènements.

Avant l'intervention, le REX dans l'entreprise était centré sur les évènements significatifs définis dans la réglementation sur la sécurité des tunnels routiers (incendie, accidents corporels et mortels et tout évènement ayant généré une fermeture de tunnels non programmée). Les analyses d'évènements étaient réalisées à chaud par les superviseurs PC, sur la base d'une procédure définie en interne. Ces analyses, en fonction du degré de gravité de l'évènement, pouvaient être complétées par un ou plusieurs débriefings :

- interne(s), piloté(s) par les cadres de l'exploitation et incluant les chefs d'équipe PC et patrouilleurs,

- plus ouvert(s), incluant potentiellement les pompiers, les services de secours et les représentants des forces de l'ordre.

Ces débriefings offraient l'occasion aux différents acteurs présents de développer une connaissance mutuelle et d'échanger sur leurs pratiques de gestion d'évènement. Cependant, en interne, les débriefings concernaient essentiellement la hiérarchie de l'exploitation (chefs d'équipes, cadres, direction). Les comptes rendus n'étaient pas toujours diffusés auprès des opérationnels et des autres services concernés par la gestion de la sécurité, tels que le service maintenance. Les analyses et les débriefings aboutissaient souvent à la définition de nouvelles règles, internes ou partagées avec les partenaires extérieurs, modifiant les procédures de gestion d'évènement existantes. Mais pour les acteurs de terrain, les liens entre l'évènement et les évolutions de procédure n'étaient pas forcément visibles. De son côté, la maintenance avait développé ses propres méthodes d'analyse d'incidents technique ou organisationnel la concernant. Ces méthodes ne portaient pas le nom de REX et n'étaient pas partagées avec les acteurs de l'exploitation.

Les enjeux de l'intervention étaient l'amélioration de la maîtrise des risques en développant les compétences des acteurs, aux niveaux individuel et collectif, d'anticipation et de gestion des évènements de toute nature, et l'enrichissement du REX pour qu'il permette de traiter d'avantage les difficultés réelles rencontrées par les opérateurs, de prendre en compte les signaux faibles et le travail collectif inter-métiers de gestion de la sécurité. 


\subsection{Objectif et hypothèses de l'intervenante : développer les ressources des acteurs pour améliorer la gestion collective de la sécurité}

Notre objectif initial était de construire une intervention capacitante pour développer les ressources des acteurs et la gestion de la sécurité. Nous soutenions une approche constructive du REX centrée sur la gestion de la sécurité au quotidien et le travail collectif inter-métiers. Nous envisagions le REX comme un processus réflexif sur les pratiques de terrain, porteur d'apprentissage collectif, de (re)construction de règles et d'évolution des pratiques.

Quatre hypothèses principales ont émergé dès le début de l'intervention qui ont structuré notre démarche :

- Le dispositif de REX de l'entreprise, centré sur les évènements «significatifs » au sens de la réglementation (les évènements majeurs, cf.2.2.), ne prend pas en compte les petits incidents et les perturbations gérés au quotidien par les opérateurs;

- Or, les situations critiques et complexes constituent des occasions essentielles d'apprentissage pour les opérateurs, pour développer leur capacité à anticiper et agir collectivement en cas d'évènement ;

- Cette capacité à gérer la sécurité se joue dans les collectifs-métiers mais aussi en intermétiers, notamment entre acteurs de supervision et intervenants de terrain ;

- La mise place des espaces de débat sur le travail peut favoriser l'évolution des pratiques et des règles d'action collectives de façon continue.

Ces quatre hypothèses ont conduit l'intervenante à élaborer une stratégie d'intervention centrée sur la création d'espaces de débat sur le travail et sur une démarche participative de conception du dispositif de REX, en partant de l'analyse du travail collectif intermétiers de gestion de la sécurité.

\subsection{Une démarche d'intervention intégrant des espaces de débat}

La méthodologie mise en œuvre en phase initiale de l'intervention correspond à une démarche d'intervention classique en ergonomie reposant dans un premier temps sur une phase d'analyse et dans un second temps sur une démarche participative de conception organisationnelle.

\subsubsection{La démarche d'analyse}

Les hypothèses sous-jacentes de l'intervention (cf. 2.1) ont structuré notre approche méthodologique, notamment en ce qui concerne la phase d'analyse et de construction du diagnostic (cf. Tableau 1).

Tableau 1 : des hypothèses aux éléments de méthodologie. Table 1: From hypotheses to aspects of methodology

\begin{tabular}{|l|l}
\hline Hypothèses & Éléments de méthodologie
\end{tabular}




\begin{tabular}{|c|c|}
\hline $\begin{array}{l}\text { Le dispositif de REX de l'entreprise, centré sur } \\
\text { les évènements "significatifs" au sens de la } \\
\text { réglementation (cf.2.2.), ne prend pas en } \\
\text { compte les petits incidents et les perturbations } \\
\text { gérés au quotidien par les opérateurs; }\end{array}$ & $\begin{array}{l}\text { Analyser les processus et les dossiers de REX } \\
\text { pour connaître les objets du REX existant ; }\end{array}$ \\
\hline $\begin{array}{l}\text { Les situations critiques et complexes } \\
\text { constituent des occasions essentielles } \\
\text { d'apprentissage pour les opérateurs pour } \\
\text { développer leur capacité à anticiper et agir } \\
\text { collectivement en cas d'évènement; }\end{array}$ & $\begin{array}{l}\text { Analyser l'activité collective quotidienne, } \\
\text { notamment lors de la gestion des situations } \\
\text { critiques (aléas et perturbations) et de la } \\
\text { gestion d'évènement, sous l'angle des } \\
\text { apprentissages collectifs: } \\
\text { - avec les superviseurs PC, les patrouilleurs et } \\
\text { les techniciens de maintenance; }\end{array}$ \\
\hline $\begin{array}{l}\text { Cette capacité à gérer la sécurité se joue dans } \\
\text { les collectifs-métiers mais aussi en inter- } \\
\text { métiers, notamment entre acteurs de } \\
\text { supervision et intervenants de terrain; }\end{array}$ & $\begin{array}{l}\text { Analyser le travail sous l'angle spécifique du } \\
\text { collectif et de la coopération (processus } \\
\text { collectifs) aux niveaux métiers et inter- } \\
\text { métiers; }\end{array}$ \\
\hline $\begin{array}{l}\text { La mise place des espaces de débat sur le travail } \\
\text { peut favoriser l'évolution des pratiques et des } \\
\text { règles d'action collectives de façon continue. }\end{array}$ & $\begin{array}{l}\text { Organiser des espaces de débat sur le travail } \\
\text { de gestion de la sécurité et analyser les } \\
\text { résultats produits dans ces espaces, en termes } \\
\text { de partage d'expérience, d'apprentissage et } \\
\text { d'évolution des règles d'action. }\end{array}$ \\
\hline
\end{tabular}

31 Ainsi, la démarche d'analyse s'est divisée en deux parties :

- L'analyse du dispositif et des dossiers de REX existants ;

- L'analyse du travail des opérateurs des trois métiers, centrée sur le travail collectif ;

L'analyse du REX a consisté essentiellement en une analyse documentaire concernant 110 dossiers de REX, les procédures et les outils structurant les pratiques de REX. Cette analyse documentaire a été complétée par une analyse de 11 réunions de débriefings après évènements ayant eu lieu pendant la période d'observations dans l'entreprise.

33 L'analyse du travail a porté sur 180 heures d'observation du travail avec les acteurs des 3 métiers qui gèrent la sécurité sur le terrain (superviseurs PC, patrouilleurs et techniciens de maintenance), complétées par 22 entretiens au fil de l'eau. Ces observations se sont déroulées sur les différents postes (matin, après-midi, nuit). Une attention particulière a été portée aux observations lors de la survenue d'un évènement. Celles-ci ont eu lieu essentiellement depuis le PC et ont été réalisées de façon continue jusqu'à la fin de l'évènement lorsque le trafic revient à la normale (excepté pour les évènements dont la gestion s'est déroulée sur plusieurs jours). Ces observations sur évènement ont été systématiquement complétées par des entretiens avec les protagonistes, en particulier avec ceux qui n'ont pu être observés en direct. L'objectif des entretiens dans ce cas était de recueillir les différentes versions du déroulement de l'évènement pour les confronter entre elles et aux informations recueillies en direct ou via la vidéo lors des observations au PC. Des observations systématiques ciblées ont aussi été conduites lors des temps de travail réflexif collectif existants dans les équipes tels que les REX informels de terrain, les relèves de poste, les réunions d'organisation interéquipes. 


\section{a) Le diagnostic REX mettant en lumière les cloisonnements entre métiers et entre} processus formels et informels

L'analyse des processus de REX et les observations du travail ont permis d'établir un diagnostic du fonctionnement du REX en lien avec la gestion de la sécurité sur le terrain, qui met en lumière des cloisonnements importants: un cloisonnement entre le REX formel et les pratiques réflexives collectives informelles, mais aussi un cloisonnement entre le REX des superviseurs PC et des patrouilleurs d'une part, et celui de la maintenance d'autre part (cf. Figure 2).

Figure 1 : Schéma synthétique des différentes formes de REX existants dans l'entreprise (cf. Casse, Caroly, \& Willmann, 2016).

Figure 1: Synthesis diagram of different forms of feedback that exist within the company
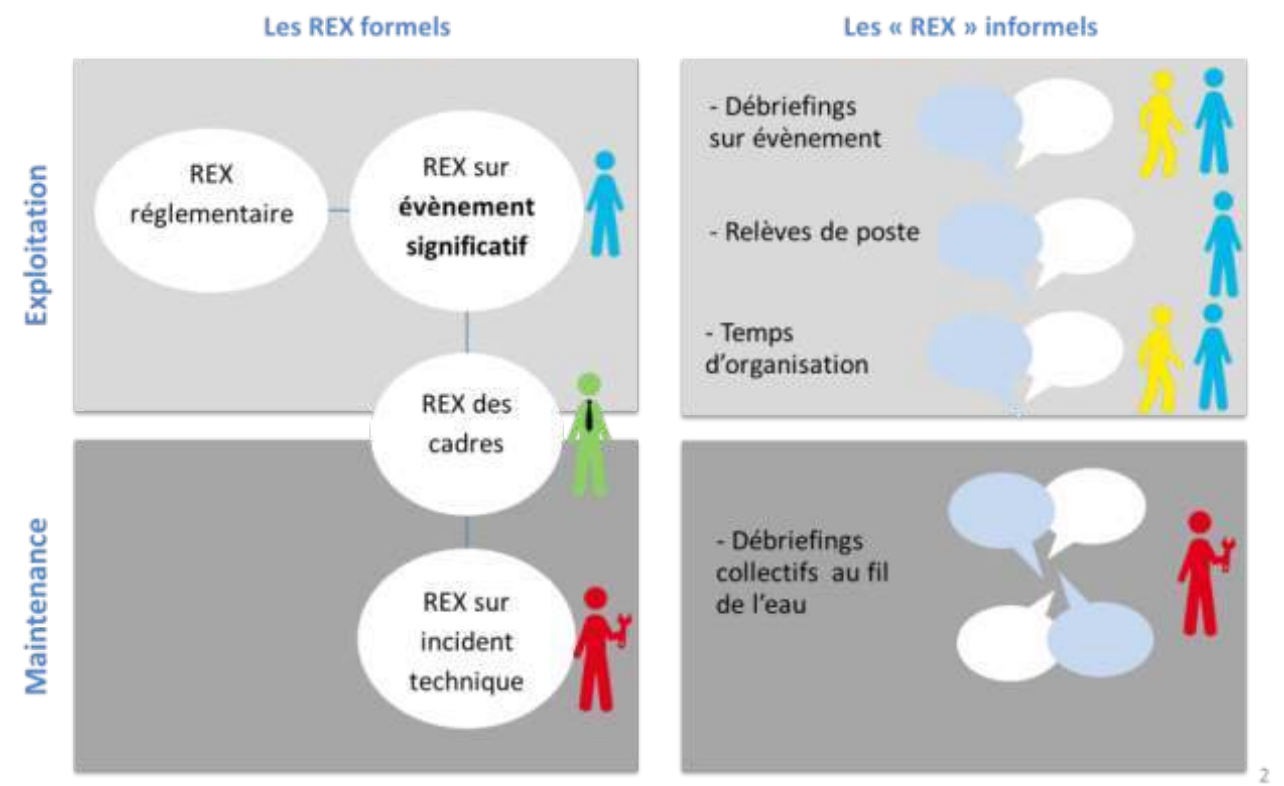

Le schéma ci-dessus a été conçu pour synthétiser et représenter le diagnostic concernant les cloisonnements observés. Il a fait l'objet d'une présentation à l'ensemble des acteurs lors des restitutions avec 3 buts principaux :

- mettre en lumière les cloisonnements pour les discuter et construire les articulations entre les secteurs cloisonnés ;

- sensibiliser les acteurs, et en particulier les managers à l'importance des pratiques informelles et leur efficacité ;

- valoriser la capacité d'auto-organisation et d'apprentissage collectif des acteurs de terrain ;

Le diagnostic a aussi mis en évidence le fait que les opérateurs de terrain désignent comme critique des incidents qui n'entrent pas dans la définition des «incidents significatifs » au sens de la réglementation, mais qui présentent cependant des problématiques complexes à gérer sur le terrain. Il a fait émerger des facteurs de criticité récurrents pour eux tels les comportements des usagers, la gestion des sous-traitants et le caractère dynamique des évènements. 


\section{b) Des restitutions avec tous les acteurs à des moments charnières de l'intervention}

37 Des restitutions des résultats de l'intervention ont été organisées tout au long de l'intervention avec les acteurs de terrain et l'encadrement (cf. Figure 2) afin de les mettre en débat au fur et à mesure de leur production. Deux restitutions auprès des opérationnels ont été réalisées (en trois réunions chacune à cause des contraintes de programmation de réunion avec des travailleurs postés), ainsi que deux restitutions auprès des cadres de l'entreprise alternativement.

Figure 2 : Synthèse des restitutions et des espaces de débat expérimentés. Figure 2: Summary of experimented restitutions and debate spaces

\begin{tabular}{|c|c|c|c|}
\hline \multirow{3}{*}{$\begin{array}{l}\text { Sessions } \\
\text { Opérationnels }\end{array}$} & $\begin{array}{l}\text { Restitution diagnostic REX } \\
\text { Proposition démarche } \\
\text { PC-patrouilleurs-TM }\end{array}$ & $\begin{array}{l}\text { Restitution diagnostic } \\
\text { gestion de la sécurité } \\
P C \text {-patrouilleurs-TM }\end{array}$ & $\begin{array}{l}\text { Restitution résultats de la } \\
\text { démarche REX } \\
P C \text {-patrouilleurs-TM }\end{array}$ \\
\hline & $\begin{array}{l}\text { Espace de débat basé sur } \\
\text { des situations critiques } \\
\text { PC-patrouilleurs-TM }\end{array}$ & $\begin{array}{l}\text { Espace de débat basé sur la } \\
\text { simulation d'évènement } \\
P C \text {-patrouilleurs }\end{array}$ & $\begin{array}{l}\text { Espace de débat basé sur } \\
\text { la simulation de REX } \\
\text { PC-patrouilleurs }\end{array}$ \\
\hline & Octobre 2012 & Avril 2013 & Octobre 2013 \\
\hline & Décembre 2012 & \multicolumn{2}{|l|}{ Juin 2013} \\
\hline $\begin{array}{l}\text { Sessions } \\
\text { Cadres }\end{array}$ & $\begin{array}{l}\text { Diagnostic REX } \\
\text { Proposition de } \\
\text { démarche } \\
\text { participative }\end{array}$ & \multicolumn{2}{|c|}{$\begin{array}{l}\text { Diagnostic gestion } \\
\text { collective de la } \\
\text { sécurité } \\
\text { Analyse des incidents } \\
\text { critiques }\end{array}$} \\
\hline Légende : & Restitutions & \multicolumn{2}{|l|}{ Espaces de débat } \\
\hline & : superviseurs PC & TM : technicien de mainte & ance \\
\hline
\end{tabular}

L'objectif des restitutions était de partager et de mettre en débat le diagnostic sur des points clés tels que le fonctionnement cloisonné du REX formel, les problèmes de diffusion des enseignements, la dynamique de REX informel. Elles ont aussi constitué des occasions pour présenter et discuter les propositions de scénarios d'organisation du REX produites par le groupe de travail et le groupe de pilotage. Pour les restitutions avec les opérationnels il s'agissait aussi d'expérimenter les modes de débat développés avec le groupe de travail sur les situations critiques inter-métiers de toute nature.

\subsubsection{La démarche participative de conception du nouveau REX}

39 La phase d'analyse a été complétée par une démarche de conception collective d'une nouvelle organisation du REX. Notre démarche de conception visait à faire dialoguer les acteurs de terrain et les cadres de l'exploitation pour aboutir à une organisation qui leur permette de s'approprier, de faire vivre et évoluer le dispositif de REX en interne.

\section{a) Structure participative}

Nous avons ainsi constitué deux instances internes : un groupe de travail inter-métiers au niveau opérationnel et un comité de pilotage au niveau encadrement. Le groupe de travail inter-métiers engageait un superviseur du PC, un patrouilleur, un technicien de maintenance, ainsi que les chefs d'équipe du PC et des patrouilleurs, tous volontaires. Le comité de pilotage (copil) était composé des cadres de l'exploitation assurant la fonction 
d'astreinte direction pour gérer les évènements en périodes de nuit, de week-ends, et les jours fériés (directeur d'exploitation, responsables maintenance, travaux et rénovation, commercial, sécurité-trafic). Les chefs d'équipe, en tant qu'acteurs pivot entre le terrain et l'encadrement supérieur faisaient partie des deux entités et avaient une fonction de « traducteurs ", partagée avec l'intervenante. Nous avons aussi associé le comité de suivi de l'intervention constitué de la direction de l'entreprise et des partenaires du projet (CETU, université) afin d'élargir la problématique interne et d'accompagner les dirigeants de l'entreprise et les institutionnels dans une conception nouvelle du REX pour les exploitants de tunnels.

\section{b) L'organisation de la démarche : alternance des instances pour faire circuler les informations et les points de vue}

41 Les réunions des trois instances impliquées dans l'intervention (groupe de travail, comité de pilotage, comité de suivi) ont été organisées en alternance de façon à permettre la circulation et la discussion des idées et des propositions d'une instance à l'autre. Le nouveau REX a été conçu dans un processus itératif d'interaction entre les 3 groupes d'acteurs. Les itérations entre le groupe de travail et le copil étaient assez rapprochées. Elles ont permis de travailler sur des scénarios communs, de partager les solutions envisagées par chaque groupe, d'aborder les outils du REX à développer, notamment la définition des objets du REX (c'est-à-dire des évènements déclencheurs de REX). Cette organisation des réunions a eu une importance pour mettre au travail les différents acteurs vis-à-vis de la gestion de la sécurité au quotidien et du REX. Par contre, elle n'a pas forcément facilité la diffusion des échanges et des avancées vers les acteurs non impliqués directement dans la démarche, qu'ils soient opérateurs ou cadres.

\subsubsection{Le dispositif d'espaces de débat inter-métiers}

\section{a) Démarche exploratoire en groupe de travail}

Une phase préparatoire en groupe de travail a été consacrée à organiser des débats collectifs à partir de situations de référence considérées comme caractéristiques de l'activité collective de gestion de la sécurité pour chaque métier ou entre les métiers. Ces situations avaient été choisies par l'intervenante à l'issue de l'analyse de l'activité. Elles concernaient à la fois des situations d'évènement «significatif» défini dans la réglementation des tunnels routiers et des situations de perturbation courante apparues comme critiques lors des observations. L'objectif était d'expérimenter des espaces de débat (Detchessahar, 2013; Rocha, 2014) qui ait une fonction « laboratoire». Les situations d'évènement comme de perturbation courante abordées dans le groupe de travail ont permis de débattre sur les règles et pratiques de terrain et leur évolution, les difficultés d'application éventuelles et sur des solutions inter-métiers. Cette démarche expérimentée en petit groupe devait permettre de former les acteurs du groupe au débat inter-métiers, de valider l'intérêt de ces espaces pour faire évoluer les règles et transformer le travail sur le terrain. À l'issue de cette expérimentation préparatoire, les participants du groupe ont sélectionné les situations critiques porteuses d'enseignements qui seraient support des expérimentations élargies. 
43 Le deuxième volet de la démarche a consisté à construire un dispositif pour déployer l'expérimentation des espaces de débat à l'ensemble des acteurs opérationnels de l'entreprise, validé en comité de pilotage.

\section{b) Expérimentations avec tous les acteurs opérationnels}

inter-métiers à l'ensemble des acteurs de la gestion de la sécurité de l'entreprise (superviseurs, patrouilleurs, TM). Nous avons testé différentes formes d'espaces de débat adaptées aux problématiques concrètes rencontrées par les opérateurs sur le terrain. Ces espaces ont porté sur des situations critiques concernant les 3 métiers impliqués, sélectionnées à l'issue de la phase exploratoire avec le groupe de travail. Nous avons cherché quelle méthode serait la plus adaptée pour «convoquer » l'activité » dans les espaces élargis (de 10 à 15 personnes) et favoriser le débat sur les difficultés de terrain rencontrées par les acteurs de la gestion de la sécurité.

Nous avons choisi d'utiliser la simulation à partir de scénarios d'évènements passés pour aider les opérateurs à se mettre en situation collectivement et susciter la confrontation des stratégies, des pratiques et le débat. Trois sessions de travail intermétiers ont ainsi été mises en place, sur la base de simulations d'évènement de plus ou moins grande importance.

La session que nous aborderons dans cet article s'est basée sur deux scénarios d'incendie. Le scénario 1 mettait en scène un évènement significatif (au sens de la réglementation) qui se déroulait en tunnel aux heures de fort trafic. Le scénario 2 concernait un incendie mineur considéré comme une situation critique, car il avait lieu en dehors des tunnels sur le bas-côté des voies circulées, la nuit. Ces deux situations d'évènements ont été sélectionnées pour leur dimension pédagogique par l'intervenante et le chef d'équipe du PC qui ont participé à l'ensemble de la démarche avec le groupe de travail. Les scénarios ont été bâtis par le chef d'équipe du PC. Les informations relatives aux dates et acteurs de l'évènement étaient neutralisées afin de les rendre " anonymes ». Des supports de simulation ont été construits à partir de photos, de plans de situations et d'informations de contexte destinés à aider les opérateurs à se projeter dans la situation. Un support écrit de recueil d'information a aussi été élaboré dans lequel des questions étaient posées à chaque opérateur-métier pour récolter les décisions et les actions de chaque acteur à chaque étape de la simulation.

Chaque session se déroulait de la même manière. Le chef d'équipe du PC préparait et animait la session. Des groupes inter-métiers étaient constitués, mélangeant des anciens et des novices. Pour chaque simulation, le chef d'équipe présentait le scénario d'évènements, ensuite il donnait les consignes pour le recueil d'information. Chaque groupe discutait ensemble et un représentant de chaque métier prenait des notes des décisions et actions. Une fois que tous les groupes avaient fait leur simulation, le chef d'équipe demandait à un groupe de présenter sa stratégie, son analyse de la situation et ses actions. Les autres groupes discutaient et commentaient la stratégie présentée et éventuellement donnaient leur propre analyse de la situation et leur stratégie. Le chef d'équipe intervenait régulièrement pour interroger les uns ou les autres sur les motivations de leurs décisions ou actions et pour préciser des règles importantes, notamment en matière de sécurité. En fin de session, les débats étaient synthétisés par le 
chef d'équipe rappelant les points de divergence qui avaient émergé et les règles importantes qui avaient été discutées, rappelées ou élaborées pendant la session.

\subsection{Retournements imprévus de situation} des décisions managériales, qui ont bouleversé le contexte des régulations collectives pour les différents métiers impliqués. Le changement le plus déterminant a eu lieu début 2013, au moment du démarrage de la démarche de conception du nouveau REX (cf. 2.4.2). Ce changement concerne la désynchronisation des équipes inter-métiers postés en 3x8 qui étaient établies depuis plusieurs années et l'introduction de la modulation dans les cycles de postes des nouveaux arrivants au PC et chez les patrouilleurs. Jusqu'ici, les équipes inter-métiers composées chacune d'une équipe fixe de patrouilleurs, d'un superviseur PC et d'une équipe fixe de péagers travaillaient sur les mêmes cycles de postes toute l'année. La désynchronisation et la modulation des cycles de poste a eu pour conséquence que les agents ont été amenés à travailler avec des collègues différents presque chaque jour, alors qu'auparavant ils fonctionnaient avec les mêmes collègues tout au long de l'année, et ce pendant plusieurs années. Les nouveaux patrouilleurs sont par ailleurs embauchés avec des contrats de polyvalents (patrouilleur-péager). Ils sont moins rémunérés que par le passé et aussi moins formés. Ces changements ont impacté le fonctionnement collectif des superviseurs et des patrouilleurs. plurielles. D'abord, les collectifs inter-métiers de superviseur et patrouilleurs ne peuvent plus s'appuyer sur des référentiels communs construits de longue date et doivent reconstruire les règles de travail à chaque changement d'équipe c'est-à-dire potentiellement chaque changement de poste. Le contenu de la formation des novices et leur connaissance du métier sont remis en cause par les anciens, et des difficultés réelles d'intégration des novices se font sentir dans les équipes.

Ces bouleversements ont posé un enjeu particulier dans l'expérimentation des espaces de débat. Dans ce contexte de déstructuration des collectifs de terrain et de perte de compétences reconnue par les opérateurs eux-mêmes et par l'encadrement, ils sont apparus comme des moyens pour développer l'apprentissage et la construction de l'expérience au niveau individuel et collectif, pour former les novices, mais aussi potentiellement construire une dynamique collective nouvelle.

\subsection{Des résultats constructifs malgré des freins socio- organisationnels}

51 La démarche d'intervention mise en œuvre a abouti à des résultats significatifs montrant la contribution effective des espaces de débat à l'enrichissement du REX et au développement des acteurs. Cependant les renversements présentés plus haut ont perturbé la dynamique d'acteurs et le contexte socio-organisationnel a limité la portée des résultats. 


\subsubsection{La dynamique d'acteurs impactée par les décisions managériales}

52 La construction sociale de l'intervention a été impactée par les changements organisationnels survenus au cours de l'intervention (cf. 2.5.). Les membres du groupe de travail étaient tous des opérateurs très actifs et impliqués dans l'entreprise, ayant de l'expérience et bénéficiant de la reconnaissance de leurs pairs (l'un était formateur des novices, un autre membre du CHSCT). Ils ont accepté de participer à la démarche avec l'espoir d'améliorer le fonctionnement du REX, qu'ils connaissaient bien et dont ils mesuraient les limites. Ils avaient, pour deux d'entre eux au moins, contribué à la mise en place du dispositif existant. Ils ont été très actifs dans la première période de travail en groupe, centrée sur l'expérimentation de nouveaux espaces de débat à partir des évènements passés et des situations critiques. Le premier bilan fait avec le groupe à l'issue de la première étape est positif en termes d'apprentissage et de souhait de continuer la démarche. Les propositions des différents membres du groupe dans la phase de réalisation des scénarios attestent de leur engagement réel dans la démarche de transformation organisationnelle. Cependant, le changement de direction et la nouvelle organisation mise en place ont eu un impact négatif sur le fonctionnement du groupe et l'implication des acteurs. Les résultats du deuxième bilan fait avec les membres du groupe à la fin de la démarche participative étaient très différents du 1er. Le superviseur, l'un des plus anciens et directement impliqué dans la conception du REX existant, avait démissionné de l'entreprise. Le patrouilleur et le technicien de maintenance ont affirmé, au moment de l'évaluation, qu'ils ont apprécié la démarche en groupe mais que pour des raisons personnelles liées aux changements en cours dans l'entreprise, ils ne désiraient plus s'impliquer dans la mise en œuvre du nouveau dispositif, alors même que nous avions collectivement envisagé qu'ils restent un groupe d'experts internes du REX et que la direction avait validé cette proposition (après discussion en comité de suivi). Les retournements du contexte de l'intervention ont donc fragilisé la construction sociale de l'intervention.

\subsubsection{Un éco-système organisationnel qui limite la portée des résultats}

53 La démarche de conception du nouveau dispositif de REX n'a pas abouti non plus en interne au résultat escompté. L'artefact conçu lors de la démarche participative (cf. 2.5.1) n'a pas pu être mis en place intégralement au sein de l'entreprise du fait d'un contexte socio-organisationnel qui a contrecarré les objectifs initiaux de la démarche.

54 Ce n'est finalement pas le dispositif conçu en groupe qui a été formalisé dans la nouvelle procédure de REX et mis en place concrètement dans l'entreprise. Suite à une volonté externe d'uniformisation des procédures entre les deux structures d'exploitation appartenant au même Maître d'ouvrage, une partie des éléments de ce dispositif a été modifiée ou supprimée. Par exemple, la valorisation des espaces de travail informels n'a pas été intégrée dans la procédure interne finale. Le dispositif formalisé dans la nouvelle procédure de l'entreprise correspond plus à un dispositif de REX standard même si les définitions y sont plus larges.

55 Par contre le dispositif développé lors de l'intervention a été formalisé de façon complète dans un document générique construit avec le CETU, "L'organisation du retour d'expérience chez les exploitants de tunnels routiers ", qui pourrait servir de base à 
l'élaboration d'une note d'information du CETU sur l'organisation du retour d'expérience chez les exploitants de tunnels routiers, diffusée au niveau national.

Le contexte structurel et social de l'entreprise a donc perturbé aussi le processus de conception participative. On peut donc conclure que l'écosystème organisationnel de l'entreprise (Detchessahar, 2011; cf. 1.2.) n'a pas favorisé la mise en place d'une organisation capacitante.

\subsubsection{Une réelle contribution des espaces de débat à l'enrichissement du REX et au développement des individus et de l'organisation}

Malgré un contexte socio-organisationnel contraignant, l'intervention a produit des résultats constructifs en lien avec la mise en place des espaces de débat sur le travail et la démarche participative de conception du nouveau REX. Particulièrement les espaces de débat basés sur la simulation d'évènements ont abouti aux résultats escomptés en matière de transmissions de connaissance, d'apprentissages et de construction de règles d'action (Casse \& Caroly, 2016).

Les échanges lors des simulations ont permis des transmissions de connaissance sur les pratiques de terrain et concernant les procédures et les règles, notamment entre les anciens et les novices. Les simulations ont mis en lumière la diversité des diagnostics, des règles mises en œuvre sur le terrain et des stratégies des opérateurs, superviseurs ou patrouilleurs. Par exemple, à l'issue de la simulation du premier scénario d'incendie (cf. 2.4.3) nous avons constaté que les 9 groupes qui ont participé à la simulation ont tous élaboré des stratégies différentes, alors que l'ensemble des opérateurs avait l'expérience de gestion d'évènements. Ainsi, les participants aux sessions de simulation ont été confrontés aux différences d'interprétation et de décision d'action entre les groupes. Ces différences ont été source de nombreuses discussions et ont permis à chacun d'élargir ses représentations, ses connaissances et potentiellement son champ d'action et de compétences.

La simulation de gestion d'incendie en tunnel (scénario 2) a permis aux novices et aux polyvalents, qui sont moins présents sur le terrain que les autres, de revoir les procédures d'intervention en cas d'évènement. Lors de cette simulation, le chef d'équipe a demandé à des novices d'expliciter comment ils ont choisi d'intervenir et le collectif a réagi lorsque les propositions d'action n'étaient pas adaptées ou pas conformes aux règles, ce qui a permis de reprendre et discuter les règles avec eux. Dans un des groupes, par exemple, un jeune polyvalent interrogé sur sa stratégie d'action ne connait visiblement pas la procédure de gestion de bouchon "dynamique » adaptée dans ce type de situation. Un patrouilleur expérimenté lui donne spontanément les principes de la répartition des patrouilleurs sur le terrain. On assiste alors à une transmission horizontale, qui en plus de permettre au novice d'apprendre et à l'ancien de faire valoir l'expérience acquise, a l'intérêt de renforcer les liens sociaux entre les anciens-fixes et les nouveaux-polyvalents.

Dans la simulation du scénario 2, au contraire, qui mettait en lumière les difficultés d'intervention des patrouilleurs en cas de congestion importante du trafic aux heures de pointe, ce fut l'occasion pour un novice d'être valorisé. En échangeant sur les pratiques d'itinéraires de chacun, le novice apporte des solutions qui ne sont pas connues de ses collègues. C'est l'occasion pour lui d'être valorisé et pour les autres d'intégrer une nouvelle option possible. 
61 Les espaces de débat ont aussi favorisé l'élaboration de règles collectives informelles et formelles et ont ainsi généré des apprentissages organisationnels. Par exemple, dans le scénario 2 survenant en période de forte congestion de trafic, les échanges entre patrouilleurs et superviseurs sur leurs pratiques ont permis de faire évoluer les règles. Les débats à partir de la simulation ont en effet fait apparaitre l'intérêt de développer une pratique normalement mise en cuvre uniquement dans des circonstances exceptionnelles, car elle comporte un danger, c'est l'intervention à contre-sens dans le tunnel. En situation de congestion, certaines équipes ont pris l'habitude de la demander systématiquement lorsqu'elles sont bloquées. D'autres n'y ont pas recours, ayant gardé le principe d'une pratique exceptionnelle. Après discussion, étant donné les tendances d'évolutions du trafic et l'augmentation de la fréquence des situations de blocage des patrouilleurs qui ont un coût certain pour l'entreprise, le chef d'équipe valide que cette pratique peut être utilisée en cas de congestion, sous réserve que le cadre d'astreinte donne son accord.

Les managers ont animé les débats sur le travail, assuré les régulations quand le collectif était bloqué et relayé les questions et les décisions au niveau de l'encadrement supérieur. Ils ont aussi parfois rappelé les règles lorsqu'ils le jugeaient nécessaire mais toujours à l'issue des discussions collectives. Par exemple, le chef d'équipe est intervenu sur un point central de gestion des incendies - la fermeture des accès dans la zone de l'incendie - alors que deux stratégies principales avaient émergé :

- la décision de fermer toutes les bretelles d'accès à l'ouvrage, y compris celles qui ne font pas partie du domaine de l'exploitant,

- ou au contraire la décision de fermer uniquement les bretelles d'accès qui sont sur le territoire de l'exploitant, considérant que les autres ne sont pas de son domaine de responsabilité.

63 Après discussion sur ces deux stratégies entre les professionnels, sans qu'une vue commune se dégage, le chef d'équipe qui animait la réunion a rappelé une valeur fondamentale du métier: la sécurité est la priorité et elle ne doit pas s'arrêter aux questions de frontières administratives ou commerciales. Ainsi, face à ce type d'évènement, il est impératif de fermer toutes les bretelles d'accès à l'ouvrage, qu'elles soient dans ou hors territoire. Une règle commune à l'ensemble des patrouilleurs a donc été partagée pour ces situations (peu fréquentes) d'évènement.

64 Ainsi les espaces de débat sur le travail ont abouti à des résultats constructifs en termes d'apprentissage individuel, collectif et organisationnel. Ils contribuent à la construction de l'expérience des opérateurs et sont source de développement. Ils permettent aussi d'enrichir le REX par une analyse approfondie des pratiques qui favorise leur évolution et celle des règles pour s'adapter à l'environnement.

65 Autre résultat positif de l'intervention, le dispositif de REX conçu en groupe de travail prend en compte les écueils du système de REX mis à jour dans le diagnostic (Casse, 2015):

- Il s'appuie sur des définitions plus larges des objets du REX, en distinguant deux catégories d'évènements pouvant déclencher des REX: les évènements déclencheurs obligatoires, correspondant aux évènements significatifs décrits dans la littérature et les évènements déclencheurs potentiels qui correspondent à "des évènements et des incidents plus courants, moins visibles mais qui constituent des signaux faibles, des indicateurs de dérives potentielles ou des précurseurs d'évènements plus importants. » Ils sont définis par des facteurs de criticité tels que: la dynamique de l'évènement, le 
comportement des usagers, les conséquences de l'évènement, les interactions avec les partenaires extérieurs (secours, prestataires...) et le contexte.

- Il implique tous les acteurs de l'exploitation et notamment les patrouilleurs qui ont été formés à faire du REX à travers les espaces de débat et la simulation. Nous avons pu constater dans ces espaces que les patrouilleurs prenaient de plus en plus d'initiatives dans les réunions, jusqu'à proposer eux-mêmes des situations critiques à analyser.

- Il formalise les deux processus de REX exploitation et maintenance et crée des passerelles entre eux. Les deux processus sont formalisés de façon complémentaire dans la procédure officielle et des étapes de diffusion des comptes-rendus et des décisions issues du REX sont prévues entre les deux services. Chaque service intègre aussi dans ses réunions d'équipe les REX liés aux autres spécialités.

- Il structure les débriefings pratiqués et redonne la place aux acteurs de terrain qui étaient un peu évacués des réunions. Trois types de débriefings sont prévus en fonction du type d'évènement (trafic, incident technique...), de sa "valeur d'enseignement » potentielle (nouveauté, gravité, rareté) et des contraintes de mobilisation des acteurs (postés) : 1-un débriefing interne avec tous les personnels concernés, pour tous les évènements significatifs, 2-un débriefing avec les partenaires externes des secours (SDIS notamment) et/ou des forces de l'ordre (CRS, gendarmes) suite aux évènements et incidents majeurs qui ont mobilisé ces acteurs et 3-un débriefing au niveau de l'encadrement (exploitation et maintenance) pour tout évènement ou incident qui pose des questions d'organisation ou de gestion spécifiques. Les débriefings sont définis comme des espaces de travail collectif, permettant à la fois de comprendre les processus passés mais aussi de construire les règles du futur. Ils ont plusieurs objectifs. Un objectif de $1^{\text {er }}$ niveau de revenir collectivement sur les processus, les stratégies d'action, les difficultés rencontrées, d'en dégager les points forts et les points faibles et d'envisager les pistes d'amélioration. Ils répondent aussi à un objectif plus global de permettre la construction progressive de référentiels partagés entre les acteurs de l'exploitation et de développer ainsi la qualité de la coopération et les compétences d'intervention collective. Les réunions de débriefing font l'objet d'un compte rendu qui permet de garder la trace des échanges et des décisions.

- Il met en valeur les espaces de travail réflexifs déjà existants dans l'entreprise, mais non valorisés jusqu'ici, tels que les temps de relève dans les équipes postées (l'analyse de l'activité collective a montré que la relève des opérateurs du PC est un lieu de partage d'expériences qui alimente le REX) et surtout les débriefings spontanés au PC entre les différents acteurs (superviseurs, patrouilleurs, techniciens, chef d'équipe). Ils sont encouragés par les responsables d'équipe, qui peuvent participer à animer les moments informels, à favoriser le partage d'expérience lors de ces échanges et demander la réalisation d'une fiche de REX s'ils le jugent pertinent.

Les résultats de l'intervention sont donc contrastés entre :

- des expérimentations qui ouvrent des perspectives très intéressantes pour renouveler la conception du REX et l'enrichir d'une dimension constructive et développementale ancrée dans le fonctionnement collectif quotidien;

- une procédure de REX qui intègre un élargissement de la démarche et du dispositif mais n'est pas tout à fait conforme aux productions collectives ;

- une construction sociale qui après une phase dynamique très constructive se termine par le désengagement des acteurs principaux.

67 L'analyse réflexive de l'intervention nous permet de discuter des conditions pour mettre en place des espaces de débat constructifs, de l'intérêt de la simulation, de la place du 
diagnostic dans l'intervention et l'évolution des compétences qu'elle entraine pour les ergonomes.

\section{Discussion et perspectives : quels apports de l'analyse de cas pour la modélisation des interventions à visée capacitante?}

\subsection{Les espaces de débat sur le travail produisent des résultats constructifs... si le contexte socio-organisationnel est favorable}

\section{testées ici :}

- des managers de proximité formés par l'ergonome au travers de la démarche participative, animaient et régulaient les débats et ont ensuite joué le rôle de relais auprès de l'encadrement supérieur pour les questions en suspens ou d'un autre niveau ;

- l'intervenante était présente lors de tous les espaces de débat, garantissant une forme de sécurité aux opérateurs dans les débats ;

- la convocation de l'activité, à travers la simulation d'évènement notamment, était structurée et outillée afin que les débats soient centrés sur le travail, les pratiques et les règles - métiers ou inter-métiers.

Cependant, l'expérimentation des espaces de débat montre aussi que ce type de dispositif, pour produire des résultats pérennes, doit s'inscrire dans un contexte de construction sociale qui soit favorable et durable. Le contexte socio-organisationnel doit permettre l'engagement des acteurs dans la durée ou le dispositif d'intervention doit intégrer tôt les possibles changements de posture des acteurs. Il doit garantir une certaine stabilité pour que les acteurs clés de la démarche, qui peuvent avoir le rôle de relais de l'intervenant dans l'entreprise sur le long terme, puissent assumer leur rôle au-delà de l'intervention. Le choix de ces acteurs et leur nombre est important pour garantir une certaine pérennité. Dans notre intervention nous avons choisi des acteurs de terrain qui avaient beaucoup d'expérience et constituaient des acteurs clés dans l'entreprise. Ces acteurs ont particulièrement mal accepté les changements imposés par la direction qui bousculaient leurs valeurs et leurs représentations du métier. Une plus grande diversité dans les profils des acteurs engagés de façon active dans la démarche (notamment en intégrant des novices / polyvalents) et un groupe un peu plus large (ils étaient 6 au départ dans le groupe de travail, puis 5) auraient peut-être permis une plus grande diversité de postures vis-à-vis du changement et la pérennité d'un groupe de référents de la démarche. La 
perspective d'une reconnaissance ou d'une valorisation de l'implication de chacun dans la démarche aurait peut-être aussi été utile.

Cette problématique est particulièrement importante en ce qui concerne les interventions menées par un intervenant externe à l'entreprise, mais elle concerne aussi les ergonomes internes. En général, celui-ci mène plusieurs missions en parallèle au sein de son entreprise, en tant qu'intervenant ou que donneur d'ordre pour des sous-traitants. Bien qu'ayant la possibilité de faire un suivi à long terme de ses interventions, il se trouve lui aussi souvent confronté à des questions de pérennité de la dynamique qu'il initie et qu'il ne peut accompagner dans la durée que de façon limitée.

\subsection{La simulation : un outil déterminant dans l'efficacité des espaces de débat}

La simulation croisée d'évènement, structurée et outillée, est une méthode efficace et constructive pour développer le débat sur le travail de gestion de la sécurité. Elle permet d'enrichir le REX d'un outil de développement. Elle fait le lien entre passé-présent et futur.

Les situations d'évènement passées servent à construire des scénarios probables, à partir desquels on amène les opérateurs à se mettre en situation, à convoquer le travail et à inventer des réponses individuelles et collectives qui serviront pour le futur. Les situations passées sont transformées en objets intermédiaires, supports de formation, d'apprentissage et outils de développement individuel, collectif et organisationnel. Grâce à la simulation et aux espaces de débat plaçant au centre le travail, le dispositif permet la construction d'expérience. Il contourne notamment les effets négatifs de l'analyse d'évènement à chaud mettant en cause les personnes. Et il articule les modes de régulations opérationnelles avec l'élaboration des règles formelles. Cette approche du REX rencontre la définition d'une organisation apprenante qui permet les processus d'apprentissage à double boucle: apprentissage direct de l'évènement et apprentissage plus global à plus long terme, à partir du travail sur les évènements de façon transversale. Il permet la construction de l'expérience collective et contribue au développement des individus et de l'organisation.

\subsection{Le diagnostic : un objet intermédiaire dans l'intervention}

74 L'analyse de cette intervention met en exergue les limites d'une démarche d'intervention sur un mode projet. La démarche d'intervention qui consiste pour l'ergonomeintervenant à produire un diagnostic à partager avec les acteurs de l'entreprise à partir duquel il va animer un processus participatif et accompagner la conception d'une nouvelle prescription (Arnoud \& Falzon, 2013a ; Barcellini et al, 2013 ; Petit, 2005 ; Rocha, 2014), ne comporte pas en elle-même les ressorts du développement des individus et de l'organisation.

Dans une dynamique de conduite de projet (Barcellini et al, 2013), éphémère par définition, l'ergonome vient avec son savoir-faire et ses outils centrés sur l'analyse du travail et le développement de l'activité, aider l'entreprise à gérer une situation de transition. Il accompagne le changement, puis il s'en va, et éventuellement revient pour évaluer et ajuster. Il fait partie d'une équipe projet ad hoc, plus ou moins élargie. Il 
implique les acteurs de terrain, qu'il accompagne pour qu'ils puissent «sortir » de leurs processus ordinaires pour entrer dans un processus de réflexion, de projection dans une activité future probable et de co-construction. Puis il laisse les acteurs retourner à leur quotidien et déployer une nouvelle activité dans la nouvelle prescription, sans qu'ils aient forcément les moyens de revenir sur cette prescription (opus cit.).

Notre étude de cas montre que cette méthodologie n'est pas adaptée à l'objectif de développer dans le long terme les compétences des individus et faire évoluer l'organisation, les pratiques et les règles de façon dynamique. L'ergonome peut dans le temps du projet initier un processus de débat, mais comme le montre notre cas, il est impératif qu'il amène d'autres méthodes pour pouvoir former et laisser la main aux acteurs internes pour que la démarche soit pérennisée (Rocha, 2014 ; Van Belleghem et al, 2015). Cela implique notamment qu'il transfère une partie de ses outils pour faire monter en compétence les acteurs de l'entreprise et en particulier les managers, en faisant confiance au fait qu'ils les utiliseront à bon escient. Cela veut dire choisir ou inventer des outils facilement transférables pour aborder le travail réel, ce qui ne semblait pas évident jusqu'ici.

77 Cela implique une évolution de la place du diagnostic ergonomique dans l'intervention. Ce n'est pas tant le diagnostic qui doit être partagé que le regard sur le travail.

\subsection{Une compétence de concepteur de dispositif d'espaces de débat}

78 L'intervenant qui initie un dispositif d'espaces de débat doit construire une dynamique qui n'est pas seulement celle de l'intervention, comme espace de transformation (du travail, de l'organisation, des représentations), délimité dans le temps, mais une dynamique de construction sur le long terme.

Dans une démarche que nous qualifierons de constructive ou de formative, l'intervenantergonome doit développer la posture et les compétences de concepteur de dispositifs de formation et de débat avec l'objectif de structurer une démarche sur le long terme qui doit fonctionner sans lui. Cette évolution passe par la transformation des représentations des ergonomes concernant la capacité des managers de l'entreprise non seulement d'accéder au travail réel et aux régulations opératives, mais aussi d'échanger sur le travail réel pour le transformer. Il s'agit notamment de confier en partie aux managers les clés de la compréhension du travail

Ces évolutions en cours dans le métier d'intervenant en ergonomie et la diversification des modes d'intervention qu'elle traduit (Van Belleghem, 2014) ouvrent des perspectives intéressantes en matière de recherche, sur les méthodes d'intervention, le métier d'ergonome et la formation notamment. 


\section{BIBLIOGRAPHIE}

Arnoud, J. (2013). Conception organisationnelle : pour des interventions capacitantes. Thèse de doctorat, Conservatoire national des arts et métiers-CNAM.

Arnoud, J., \& Falzon, P. (2013a). Changement organisationnel et reconception de l'organisation : Des ressources aux capabilités. Activités 10(2), 109-130. URL : https://activites.revues.org/760

Arnoud, J., \& Falzon, P. (2013b). La co-analyse constructive des pratiques. In P. Falzon (Ed.), Ergonomie constructive (pp. 223-236). Paris : Presses Universitaires de France.

Barcellini, F., Van Belleghem, L., \& Daniellou, F. (2013). Les projets de conception comme opportunité de développements des activités. In P. Falzon (Ed) Ergonomie constructive (191-206). Paris : PUF

Caroly, S. (2010). Activité collective et réélaboration des règles : des enjeux pour la santé au travail. HDR, Université Victor Segalen-Bordeaux II.

Casse, C. (2015). Concevoir un dispositif de retour d'expérience intégrant l'activité réflexive collective : un enjeu de sécurité dans les tunnels routiers. Thèse de doctorat, Université Grenoble-Alpes.

Casse, C., \& Caroly, S. (2016). Les espaces de débat : un levier pour faire du retour d'expérience « effectif ». Actes du $51^{\text {ème }}$ Congrès de la SELF, Marseille, 21-23/09/16.

Casse, C., Caroly, S., \& Willmann, C. (2016). Concevoir un dispositif de retour d'expérience intégré pour plus de résilience. In les actes du congrès Lambda-Mu, Institut pour la Maîtrise des Risques (SaintMalo, 11-13 octobre).

Chaudet, P. (2013). Des conditions favorables à une réflexion collective de l'expérience. Éducation permanente, 196, 53-63.

Clot, Y. (2006). Une intensification du travail peut-elle en cacher une autre ? In Ph. Askenazy et al. (Eds.), Organisation et intensité du travail (pp. 313-317). Toulouse : Octarès.

Clot, Y., Faïta, D., Fernandez, G., \& Scheller, L. (2000). Entretiens en autoconfrontation croisée : une méthode en clinique de l'activité, Pistes, 2(1), 8 p.

Dejours, C. (2009). Travail vivant. Paris : Payot.

Detchessahar, M. (2001). Le « dire » et le « faire » : Contribution à l'étude des nouvelles formes d'organisation. Revue de gestion des ressources humaines, 39, 43-56.

Detchessahar, M. (2011). Santé au travail. Revue française de gestion, 5, 89-105.

Detchessahar, M. (2013). Faire face aux risques psycho-sociaux : quelques éléments d'un management par la discussion. Négociations, $\mathrm{n}^{\circ} 19,57-80$.

Detchessahar, M., Gentil, S., Grevin, A., \& Stimec, A. (2015). Quels modes d'intervention pour soutenir la discussion sur le travail dans les organisations ? @ GRH, 3, 63-89.

Dechy, N., \& Dien, Y. (2007). Les échecs du retour d'expérience dans l'industrie : problème de verticalité et ou de transversalité ? Conférence IMdR - GRID relative à la protection contre la malveillance et l'information de gestion. (13-14 Décembre, Paris)

Falzon, P. (2013). Ergonomie constructive. PUF : Paris. 
Garrigou, A., Thibault, J-F., Jackson, M., \& Mascia F. (2001). Contributions et démarche de l'ergonomie dans les processus de conception. Perspectives interdisciplinaires sur le travail et la santé, 3-2. URL : http://pistes.revues.org/3725

Hoyrup, S., \& Elkjaer, B. (2006). Reflection: Taking it beyond the individual. In D. Boud, P. Cressey, \& P. Docherty (Eds.), Productive Reflection at Work: Learning for Changing Organizations (pp. 29-42). London: Routledge.

Maline, J. (1997). Simuler pour approcher la réalité des conditions de réalisation du travail : la gestion d'un paradoxe. In P. Béguin P., \& A. Weill-Fassina (Eds.), La simulation en ergonomie : connaître, agir et interagir (pp. 97-111). Toulouse : Octarès.

Mollo, V., \& Nascimento, A. (2013). Pratiques réflexives et développement des individus, des collectifs et des organisations. In P. Falzon (Ed.), Ergonomie constructive (pp. 207-221). PUF : Paris.

Petit, J. (2005). Organiser la continuité du service : intervention sur l'organisation d'une Mutuelle de santé . Thèse de doctorat en ergonomie, Université Victor-Ségalen - Bordeaux 2.

Petit, J., Querelle, L., \& Daniellou, F. (2007). Quelles données pour la recherche sur la pratique de l'ergonome? Le Travail Humain, 70(4), 391-411.

Rocha, R. (2014). Du silence organisationnel au développement du débat structuré sur le travail : les effets sur la sécurité et sur l'organisation. Thèse de doctorat, Université de Bordeaux.

Rocha, R., Mollo, V., \& Daniellou, F. (2015). Work debate spaces: A tool for developing a participatory safety management. Applied Ergonomics, 46, 107-114.

Rogalski, J. (1994). Formation aux activités collectives. Le Travail Humain, 57(4), 367-386.

Rogalski, J. (1995). Former à la coopération dans la gestion des sinistres : élaboration collective d'un dispositif d'actions. Éducation permanente, 123, 47-64.

Sen, A. (1993). Capability and Well-Being. In : T. Nussbaum \& A. Sen, The Quality of Life (pp. 30-52). Oxford: Clarendon Press.

Van Belleghem, L. (2014). Perspectives pour une stratégie de diversification des pratiques en ergonomie. In Diversité des interventions, diversité des populations : quels enjeux, quels défis pour l'ergonomie? Actes du 45ème congrès de l'Association Canadienne d'Ergonomie, Montréal, 7 au 9 octobre.

Van Belleghem, L. (2016). Eliciting activity: a method of analysis at the service of discussion. Le Travail Humain, 79(3), 285-305.

Van Belleghem, L., \& Forciolli Conti E. (2015). Une ingénierie de la discussion ? Chiche ! In Actes du $50^{\text {ème }}$ congrès de la SELF. Paris, 23 au 25 septembre.

\section{RÉSUMÉS}

Les ergonomes revendiquent depuis de nombreuses années l'importance de la participation de tous les acteurs et en particulier des opérateurs de terrain dans les projets de transformation organisationnelle dans les entreprises. Ils développent des approches constructives (Falzon, 2013) et se préoccupent de plus en plus de l'épanouissement des travailleurs. Ils s'appuient pour cela sur des nouvelles méthodes d'intervention telles que les espaces de débats sur le travail (Detchessahar, 2013; Rocha, Mollo, \& Daniellou, 2015) pour mettre en œuvre une approche nouvelle de l'intervention à visée capacitante. À partir d'une analyse réflexive structurée d'une intervention pour améliorer le retour d'expérience chez un exploitant de tunnels routiers, 
l'objectif de cet article est de définir en quoi et comment les espaces de débat sur le travail, comme méthode d'intervention capacitante, peuvent enrichir le retour d'expérience. Nous verrons que la démarche projet utilisée en ergonomie, basée sur un diagnostic partagé et une démarche participative de conception, ne suffit pas dans la durée pour soutenir le développement des individus et de l'organisation. Nous montrons au contraire que la mise en œuvre d'espaces de débat sur le travail produit des résultats intéressants en matière d'apprentissages individuels et organisationnels de long terme et en ce qui concerne l'amélioration du retour d'expérience. Nous discuterons ensuite des conditions et des principes pour la mise en place et l'efficacité de ces espaces de débat. Ceci permet d'entrevoir l'évolution des méthodes et des compétences de l'ergonome en lien avec le développement d'interventions capacitantes.

For several years ergonomists have been claiming that the participatory approach - especially at the coalface in organizational and spatial design projects - is vital in companies. In recent years constructive approaches have been developed by researchers and practitioners in ergonomics (Falzon, 2013). The workers' self-fulfillment is of increasing concern within companies. New intervention methods such as work debate spaces (Detchessahar, 2013; Rocha, Mollo, \& Daniellou, 2015) are used as supports to foster workers' capacities and competencies (Sen, 1993) within companies. Based on a structured feed-back on an ergonomic intervention designed to improve learning from experience with a road tunnel operator, the aim of this article is to highlight the methods and conditions for developing individual and collective capacities within the organization. It will be seen that the ergonomic project approach based on a shared diagnosis and a participative approach to design involving the workers is not enough to support the longterm development of the workers' competencies. The article demonstrates how "work debate spaces" contribute to the development of individual and organizational learning. In conclusion this article will discuss the minimal conditions for their implementation in relation to the necessary evolutions for ergonomic intervention methods and competencies.

\section{INDEX}

Mots-clés : intervention, tunnel, retour d'expérience, espaces de débat sur le travail

Keywords : intervention, tunnel, learning from experience, work debate spaces

\section{AUTEURS}

\section{CHRISTELLE CASSE}

PACTE, Institut d'études politiques, BP 48, 38040 Grenoble Cedex 9. christelle_casse@yahoo.fr

\section{SANDRINE CAROLY}

PACTE, Institut d'études politiques, BP 48, 38040 Grenoble Cedex 9. sandrine.caroly@upmfgrenoble.fr 